\title{
Ocorrência de Aprostocetus hagenowii (Hymenoptera, Eulophidae), parasitoide de ootecas da barata americana, no Rio Grande do Sul
}

\author{
Occurrence of Aprostocetus hagenowii (Hymenoptera, Eulophidae), parasitoid of the american \\ cockroach oothecae at Rio Grande do Sul
}

\author{
Marcial Corrêa Cárcamo ${ }^{{ }^{*}}$ Rosiane Kleinhans Brandão $^{\mathrm{I}}$ Valmir Antonio Costa ${ }^{\mathrm{I}, \mathrm{II}}$ \\ Paulo Bretanha Ribeiro ${ }^{\mathrm{I}}$
}

\begin{abstract}
- NOTA -
RESUMO

Relata-se a ocorrência do parasitoide Aprostocetus hagenowii (Ratzeburg, 1952) (Hymenoptera: Eulophidae) em ooteca de Periplaneta americana Linnaeus, 1758 (Blattodea: Blattidae), no extremo Sul do Brasil. As ootecas foram coletadas no mês de dezembro de 2007, no campus da Universidade

emergence of the parasitoids. The infested ootheca presented 89 parasitoids (79 females and 10 males). The referred occurrence represents the first report to southern Brazil. Knowing the regions of occurrence of the natural enemies of $\boldsymbol{P}$. americana is of great importance when developing a control strategy to the populations of the blatod.
\end{abstract}

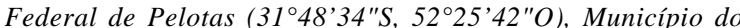
Capão do Leão, Rio Grande do Sul. Após a coleta, as ootecas foram acondicionadas individualmente em tubos de ensaio, sendo posteriormente mantidas em câmara climatizada a $25^{\circ} \mathrm{C}$, com umidade relativa $=70 \%$, até a eclosão das ninfas ou emergência dos parasitoides. Uma das ootecas estava parasitada e apresentou 89 parasitoides (79 fêmeas e 10 machos); a referida ocorrência constitui o primeiro registro para o Sul do Brasil. O conhecimento das regiões de ocorrência dos inimigos naturais de P. americana é de grande importância para se traçar uma estratégia de controle das populações desse blatódeo.

Palavras-chave: Periplaneta americana, parasitoide de ootecas, controle biológico.

\section{ABSTRACT}

It is reported the occurrence of the parasitoid Aprostocetus hagenowii (Ratzeburg, 1952) (Hymenoptera: Eulophidae) in oothecae of Periplaneta americana Linnaeus, 1758 (Blattodea: Blattidae) at the extreme southern Brazil. The oothecae were collected in December of 2007 at the campus of the Universidade Federal de Pelotas (31⒋'34"S, $52^{\circ} 25^{\prime} 42^{\prime \prime} W$ ), city of Capão do Leão, Rio Grande do Sul. After the collection the oothecae were placed individually in glass vials maintained in acclimatized chamber at $25^{\circ} \mathrm{C}$, with relative air humidity $=70 \%$ until the eclosion of the nymphs or the
Key words: Periplaneta americana, oothecae parasitoid, biological control.

Os blatódeos sinantrópicos podem atuar como transmissores e também como reservatórios de agentes patogênicos, determinando sua importância na sanidade animal e humana. Dessa forma, Periplaneta americana Linnaeus, 1758 (Blattodea, Blattidae) foi objeto de vários estudos referentes à condição de vetor de patógenos, sendo identificados diversos vírus, bactérias, fungos, protozoários e helmintos (GUTHRIE \& TINDALL, 1968; CLOAREC et al., 1992; KOPANIC et al., 1994; ZORZENON, 2002; THYSSEN et al., 2004; LEE, 1958; PÉREZ, 1989).

Essa espécie de blatódeo apresenta hábitos abscônditos (GUIMARÃES, 1984) e, segundo PINTO et al. (2007), fazem a postura de suas ootecas em esconderijos próximos aos locais com abundância de alimentos para as ninfas.

A estratégia de alternar habitats contribui para competência vetorial desse grupo, pois durante o dia repousam em ambientes escuros, úmidos e quentes,

'Programa de Pós-graduação em Parasitologia (PPGP), Universidade Federal de Pelotas (UFPel), CP 354, 96010-900, Pelotas, RS, Brasil. E-mail: marcial.carcamo@gmail.com.*Autor para correspondência.

IIInstituto Biológico, Agência Paulista de Tecnologia dos Agronegócios (IB-APTA), Centro Experimental Central do Instituto Biológico, Campinas, SP, Brasil. 
como tubulações de esgoto, fossas sépticas e latrinas. À noite, invadem habitações como depósitos de ração, armazéns, restaurantes, cozinhas e hospitais (VIANNA, 2000).

LEBECK (1991), em uma revisão sobre himenópteros, citou 18 espécies de parasitoides como inimigos naturais de baratas, e oito dessas espécies utilizam ootecas de $\boldsymbol{P}$. americana como hospedeiros para o desenvolvimento de seus ovos, suas larvas e suas pupas. O autor ainda ressaltou a importância do parasitoide gregário Aprostocetus hagenowii (Ratzeburg, 1952) (Hymenoptera, Eulophidae) para o controle dessa espécie de blatódeo.

SUITER et al. (1998) observaram uma infestação de até $100 \%$ em ootecas sentinelas, expostas um mês após a soltura inundativa do parasitoide $\boldsymbol{A}$. hagenowii no ambiente. Essas taxas mantiveram-se flutuando acima de $40 \%$ nos cinco meses após a primeira soltura, apresentando uma diminuição com a queda da temperatura ambiental.

Segundo NARASIMHAM \& SANKARAN (1979), esse parasitoide é atraído por ambientes secos, e temperaturas inferiores a $19^{\circ} \mathrm{C}$ cessam sua atividade. Apesar disso, A. hagenowii possui uma ampla distribuição mundial, já tendo sido encontrado em diferentes áreas geográficas, tais como Austrália, Índia, Arábia Saudita, Taiwan, Trinidad e Tobago, Estados Unidos da América e Japão (VARGAS \& FALLAS, 1974; YOSHIKAWA \& IKUSHIMA, 1956; ROTH \& WILLIS, 1960). No Brasil, esse parasitoide foi encontrado acometendo ootecas de P. americana no Rio de Janeiro (DE SANTIS, 1980) e no Mato Grosso do Sul (TEBALDI et al., 2003).

Este estudo teve como objetivo relatar a ocorrência de A. hagenowii parasitando ooteca de $\boldsymbol{P}$. americana no Sul do Rio Grande do Sul.

Durante o mês de dezembro de 2007, foram coletadas 28 ootecas nos prédios do Departamento de Microbiologia e Parasitologia da Universidade Federal de Pelotas (3148’34"S, 52²5’42"O). Logo após, estas foram acondicionadas individualmente em tubos de ensaio e levadas à estufa (B.O.D.), com temperatura de $25^{\circ} \mathrm{C}$ e umidade relativa do ar acima de $70 \%$; as ootecas coletadas que não apresentaram emergência e/ou eclosão após dois meses foram eliminadas. Após 22 dias da data de coleta, foi observada a emergência de 89 parasitoides de uma ooteca (79 fêmeas e 10 machos), os quais foram mortos e acondicionados em frascos contendo álcool $70 \%$.

A identificação do A. hagenowii foi realizada usando a chave proposta por GRAHAM (1987). O material coletado e identificado está depositado na
Coleção Entomológica do Departamento de Microbiologia e Parasitologia (DEMP - UFPel).

A ocorrência de A. hagenowii parasitando ootecas de $\boldsymbol{P}$. americana constitui o primeiro registro de parasitismo dessa espécie para o Rio Grande do Sul. A presença desse micro-himenóptero poderá significar uma estratégia a mais para a redução das populações desse blatódeo no Sul do Brasil, pois este apresenta algumas características que fazem dele, em teoria, um bom agente para o controle biológico inundativo de $\boldsymbol{P}$. americana; dentre essas características, estão: capacidade de parasitar até duas ootecas por fêmea (NARASIMHAM, 1984), fácil criação e manipulação em laboratório (LEBECK, 1991), pequeno tamanho e grande número de indivíduos produzidos por ooteca, havendo registro da obtenção de 261 parasitoides em um hospedeiro (ROTH \& WILLIS, 1954).

Devido a essas características, talvez a baixa prevalência de parasitismo no ambiente pudesse ser compensada por estratégias de liberação inundativa, como acontece no combate a várias outras pragas, citando-se como exemplo a traça-do-tomateiro, Tuta absoluta (Meyrick) (Lepidoptera: Gelechiidae), por Trichogramma pretiosum Riley (Hymenoptera: Trichogrammatidae) (HAJI et al., 2002); essa é uma suposição que precisaria ser confirmada com estudos no campo.

Esse parasitoide ainda acomete ootecas de outros blatódeos, dentre eles: Blatta orientalis (USMAN, 1949), Eurycotis biolleyi (VARGAS \& FALLAS, 1974), Parcoblatta spp. (EDMUNDS, 1953), Neostylogypa rhombifolia, P. australasiae (ROTH \& WILLIS, 1954), P. fuliginosa (FLEET \& FRANKIE, 1975). Isso permite que este seja usado no controle de várias espécies de blatódeos, facilitando a manutenção das populações desse himenóptero no ambiente.

\section{AGRADECIMENTOS}

À Coordenação de Aperfeiçoamento de Pessoal de Nível Superior (CAPES), pela concessão de bolsa ao pesquisador Cárcamo.

\section{REFERÊNCIAS}

CLOAREC, A. et al. Cockroaches as carriers of bacteria in multi-family dwellings. Epidemiology and Infection, v.109, p.483-490, 1992.

DE SANTIS, L. Catalogo de los Himenopteros brasilenos de la serie parasitica incluyendo Bethyloidea. Curitiba: Universidade Federal do Paraná, 1980. 395p.

EDMUNDS, L.R. Collecting and culturing of native wood roaches in Ohio, with some additional notes on their parasites. Entomological News, v.64, p.225-230, 1953. 
FLEET, R.R.; FRANKIE, G.W. Behavioural and ecological characteristics of a Eulophid egg parasite of two species of domiciliary cockroches. Enviromental Entomology, v.4, p.282-284, 1975

GRAHAM, M.W.R. de V. A reclassification of the European Tetrastichinae (Hymenoptera: Eulophidae), with a revision of certain genera. Bulletin of the British Museum, v.55, n.1, p.1-392, 1987

GUIMARÃES, J.H. Baratas: manejo integrado em áreas urbanas. São Paulo: Agroquímica Ciba Geigy, 1984. N.25

GUTHRIE, D.M.; TINDALL, A R. The biology of cockroach London and Beccles: Edward Arnold, 1968. 408p.

HAJI, F.P.N. et al. Trichogramma pretiosum para o controle de pragas no tomateiro industrial. In: PARRA, J.R.P. et al. (Eds.). Controle biológico no Brasil. Parasitóides e predadores. São Paulo: Manole, 2002. p.477-494.

KOPANIC, R.J. et al. Cockroaches as vector of Salmonella: laboratory and field trials. Journal of food protection, v.57, p.125-132, 1994

LEBECK, L.M. A review of the hymenopterous natural enemies of cockroaches with emphasis on biological control. Entomophaga, v.36, n.3, p.355-352, 1991.

LEE, D.L. Digestion in Leidynemia appendiculata (Leidy, 1850) a nematode parasitic in cockroaches. Parasitology, v.48, p.437-447, 1958 .

NARASIMHAM, A.U. Comparative studies on Tetrastichus hagenowii (Ratzeburg) and T. asthenogmus (Waterston), two primary parasites of cockroach oothecae, and on their hyperparasite Tetrastichus sp. (T. mister (Ness) group) (Hymenoptera: Eulophidae). Bulletin of Entomological Research, v.74, p.175-189, 1984

NARASIMHAM, A.U.; SANKARAN, T. Domiciliary cockroaches and their oothecal parasites in India. Entomophaga, v.24, n.3, p.273-279, 1979.

PÉREZ, J.R. La cucaracha como vector de agentes patogenos. Boletín de la Oficina Sanitaria Panamericana, v.107, n.1, p.41-53, 1989 .

PINTO, A.S. et al. Manejo de pragas urbanas. Piracicaba: CP2, 2007. 208p.
ROTH, L.M.; WILLIS, E.R. The biology of the cockroach egg parasite, Tetrastichus hagenowii, Transactions of the American Entomological Society, v.80, p.53-72, 1954

ROTH, L.M.; WILLIS, E.R. The biotic associations of cockroaches. Washington: Smithsonian Institute, 1960. p.470.

SUITER, D.R.. Seasonal incidence and biological control potential of Aprostocetus hagenowii (Hymenoptera: Eulophidae) in treehole microhabitats. Environmental Entomology, v.27, n.2, p.434-441, 1998.

TEBALDI, I.F. et al. Aprostocetus (Tetrastichodes) hagenowii (Hymenopetera: Eulophidae) atacando ootecas da barata doméstica Periplaneta americana (Blattodea, Blattidae) em Mato Grosso do Sul. In: VI CONGRESSO DE ECOLOGIA DO BRASIL, 2003, Fortaleza, CE. Anais do VI Congresso de Ecologia do Brasil. Fortaleza: Sociedade de Ecologia do Brasil, 2003. V.6, p.292-293.

THYSSEN, P.J. et al. O Papel de insetos (Blattodea, Diptera e Hymenoptera) como possíveis vetores mecânicos de helmintos em ambiente domiciliar e peridomiciliar. Cadernos de Saúde Pública, v.20, n.4, p.1096-1102, 2004. Disponível em: <http:/ / w w w. s c i e l o.b r / s c i e lo.ph p ? p i d = S 0102 311X2004000400025\&script=sci_arttext $>$. Acesso em: $20 \mathrm{Jul}$. 2008. doi: 10.1590/S0102-311X2004000400025.

USMAN, S. Some observations on the biology of Tetrastichus hagenowii Ratz. An egg parasite of the house-cockroach (Periplaneta Americana L.). Current Science, v.11, p.407408, 1949

VARGAS, M.V.; FALLAS, F.B. Notes on the biology of Tetrastichus hagenowii (Hymenoptera: Eulophidae) a parasite of cockroach oothecae. Entomology News, n.85, p.23-26, 1974.

VIANNA, E.E.S. et al. Performance reprodutiva de Periplaneta americana Linneu, 1758 (Blattodea: Blattidae). Arquivos do Instituto Biológico, v.67, n.1, p.99-107, 2000.

YOSHIKAWA, K.; IKUSHIMA, I. Some biological notes on a parasitic wasp on cockroach, Tetrastichus hagenowii Ratzeburg (Hymenoptera: Eulophidae). Medicine and biology, v.40, p.127-129, 1956.

ZORZENON, F.J. Noções sobre as principais pragas urbanas. Biológico, v.64 n.2 p.231-234, 2002. 